\title{
Polymorphisme protéique sanguin chez le poney de Mogod de Tunisie
}

\author{
A. Chabchoub ${ }^{1 *}$ Z. Mbarki ${ }^{1}$ F. Lasfar ${ }^{2}$ \\ F. Landolsi ${ }^{1}$ I. Turki ${ }^{3}$ L. Ouragh ${ }^{4}$
}

\section{Mots-clés}

Poney de Mogod - Polymorphisme biochimique - Protéine sanguine Tunisie.

\begin{abstract}
Résumé
Le poney de Mogod est originaire du nord ouest de la Tunisie. Ses aptitudes physiques lui confèrent une bonne adaptation et utilisation en montagne. Depuis le début du XXe siècle son effectif a subi une réduction considérable. Un plan de relance nécessite une meilleure caractérisation de la race, notamment d'un point de vue génétique. De plus, celle-ci permettrait de rechercher une éventuelle filiation de ce poney avec les autres chevaux de la région. Les systèmes électrophorétiques de l'albumine (Al), du composant GC (Gc), de la postalbumine $(\mathrm{Xk})$, de l'estérase basique (Es), de la transferrine ( $\mathrm{Tf}$ ) et de la préalbumine $(\mathrm{Pi})$ ont été étudiés chez 47 poneys de Mogod par la technique d'électrophorèse en gel de polyacrylamide. Les fréquences alléliques des six systèmes ont été estimées comme suit: $\mathrm{Al}^{\mathrm{A}}: 0,36 ; \mathrm{Al}^{\mathrm{B}}: 0,64 ; \mathrm{Gc}^{\mathrm{F}}: 1,00$; $\mathrm{Xk}^{\mathrm{K}}: 1,00 ; \mathrm{Es}^{\mathrm{I}}: 0,64 ; \mathrm{Es}^{\mathrm{G}}: 0,18 ; \mathrm{Es}^{\mathrm{F}}: 0,17 ; \mathrm{Es}^{\mathrm{S}}: 0,01 ; \mathrm{Tf}^{\mathrm{D}}: 0,41 ; \mathrm{Tf}^{\mathrm{F} 2}$ : 0,$31 ; \mathrm{Tf}^{\mathrm{H}}: 0,12 ; \mathrm{Pi}^{\mathrm{L}}: 0,35 ; \mathrm{Pi}^{U}: 0,22 ; \mathrm{Pi}^{\mathrm{R}} ; \mathrm{Pi}^{\mathrm{S}}: 0,09$. Une comparaison entre le polymorphisme protéique sanguin du poney de Mogod et celui des chevaux pur-sang Arabes, Barbes et Arabes-Barbes a été réalisée sur une base bibliographique.
\end{abstract}

\section{INTRODUCTION}

Dans l'extrême nord de la Tunisie, il existe une population d'équidés appelée poney Tunisien ou poney de Mogod. La région de Mogod en est le berceau (figure 1). Il est répandu dans les régions d'Amdoun, de Héllil Mogod et de Nefza (2). La production du poney a diminué fortement depuis la Seconde Guerre mondiale et l'avènement de la motoculture dans les régions forestières du nord ouest de la Tunisie (4). Il serait dommage de perdre cette race qui existe depuis des siècles en Tunisie. Les dernières statistiques estiment un nombre total aux alentours de 1000 individus (10). Un plan directeur lui est actuellement réservé pour redynamiser cette production.

Lors de précédentes études $(2,10)$, le poney de Mogod a été caractérisé morphologiquement. Il a une taille au garrot variable entre 1,2 et $1,4 \mathrm{~m}$, avec un corps bien proportionné, des muscles prononcés, une encolure courte et forte, aplatie latéralement et droite, un dos droit et voussé, et des membres courts (figure 2).

1. Service de pathologie médicale équine, Ecole vétérinaire, Sidi-Thabet 2020, Tunisie. 2. Fnarc, Sidi-Thabet 2020, Tunisie.

3. Enmv, Sidi-Thabet 2020, Tunisie.

4. Laboratoire d'analyses génétiques vétérinaires, Institut agronomique

et vétérinaire Hassan II, Rabat, Maroc.

* Auteur pour la correspondance

Tél. : +21671552200; fax : +216715524 41

E-mail : chabchoubahmed2001@yahoo.fr ; chabchoub.ahmed@iresa.agrinet.tn
Ce poney est très fort malgré sa petite taille. Il est très vif et adroit. Ses aptitudes sont considérables. Son habitat se trouve dans les montagnes et dans les forêts où il est un équidé idéal. C'est un poney rustique capable de vivre à l'état sauvage sans intervention humaine. Il était employé au nord ouest de la Tunisie pour le transport de liège, des tanins...

Des travaux précédents $(2,10)$ rapportent qu'il se rapproche plus du cheval que du poney. En effet, ses yeux, son garrot, ses reins, sa croupe montrent une grande ressemblance avec le cheval Barbe. Certaines de ses caractéristiques semblent ne pas s'appliquer parfaitement à la définition d'un poney ; il est plus haut que long, avec une longueur du canon supérieure à celle du Barbe, contrairement aux caractéristiques d'un poney (10).

Pour d'autres auteurs, le poney de Mogod constitue une race spéciale. Ce serait un cheval primitif autochtone qui se serait déplacé avec son propriétaire pour être à l'abri, dans les montagnes, des invasions successives. Cette race serait alors devenue montagnarde (4). Des hypothèses proposent qu'il s'agit d'une variété du cheval Barbe (2), Arabe-Barbe ou qu'il dériverait du cheval Arabe (1).

Le présent travail est une contribution à une meilleure connaissance de la population du poney de Mogod en Tunisie par sa caractérisation génétique. Il propose d'étudier le polymorphisme biochimique des systèmes électrophorétiques : albumine (Al), composant $\mathrm{GC}(\mathrm{Gc})$, postalbumine $(\mathrm{Xk})$, estérase basique (Es), transferrine (Tf) et préalbumine $(\mathrm{Pi})$. 


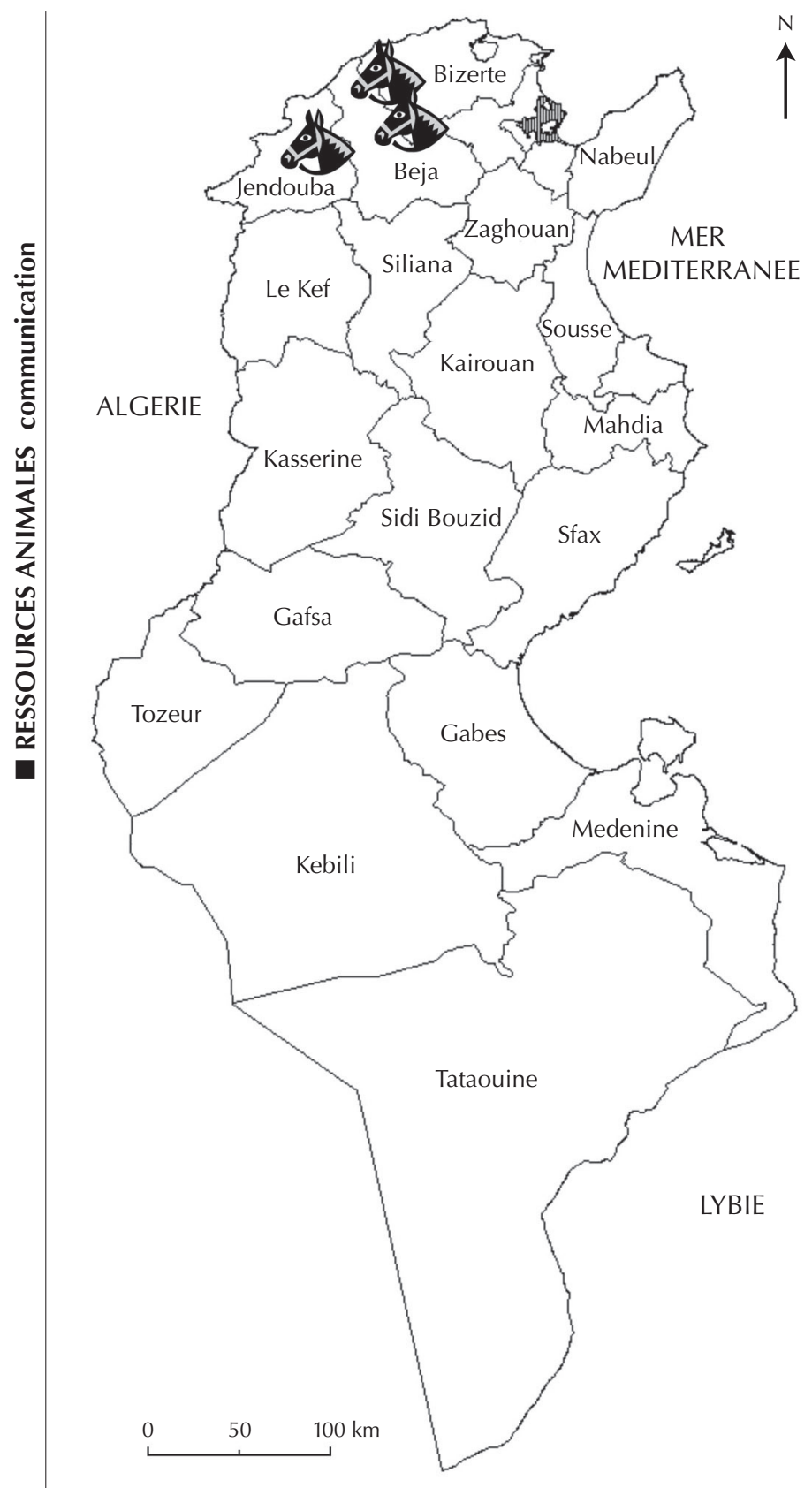

Figure 1 : carte de la Tunisie; situation géographique de la région de Mogod, berceau du poney Tunisien.

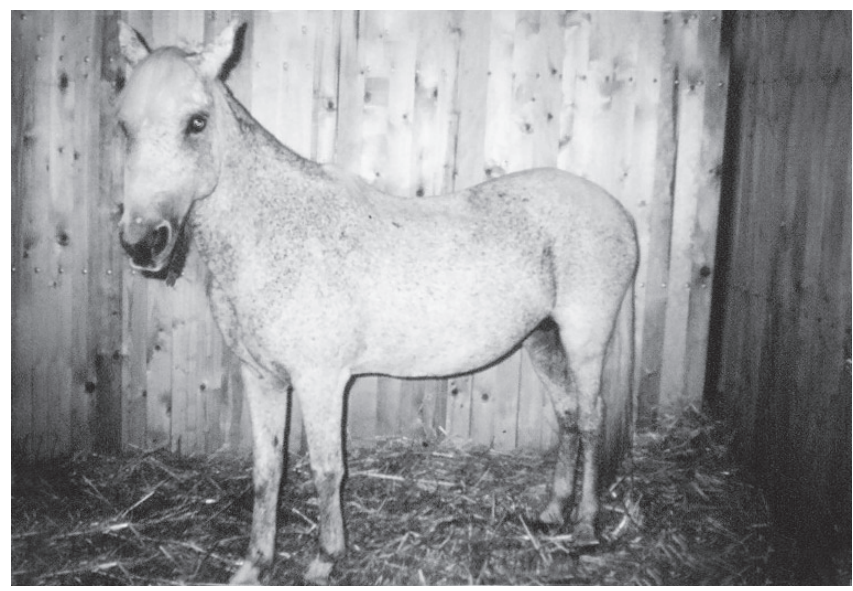

Figure 2 : le poney de Mogod.

\section{MATERIEL ET METHODES}

Les analyses ont été effectuées sur un effectif de 47 poneys de Mogod, non apparentés, âgés de 5,8 $( \pm 3,3)$ ans, comportant 12 mâles et 35 femelles, et provenant des régions du nord ouest de la Tunisie (Nefza, Amdoun, Mogod). Tous ces animaux avaient déjà fait l'objet d'une caractérisation morphométrique $(2,10)$. Le choix des animaux a été fait par tirage au sort. Les prélèvements sanguins ont été réalisés au niveau de la veine jugulaire dans des tubes secs.

La technique utilisée a été celle de l'électrophorèse en gel polyacrylamide $(\mathrm{pH}=8,9)(5)$. La concentration du gel de séparation a été de 10 p. 100 . Un courant constant de $40 \mathrm{~mA}$ par gel $(20 \mathrm{~cm} \mathrm{x}$ $20 \mathrm{~cm}$ x $0,75 \mathrm{~mm})$ a été appliqué. La tension de $300 \mathrm{~V}(15 \mathrm{~V} / \mathrm{cm})$ enregistrée au début a augmenté au cours de l'électrophorèse jusqu'à atteindre $900 \mathrm{~V}$ en fin de migration au bout de $3 \mathrm{~h} \mathrm{30}$. Chaque protéine a été codée par un gène de structure et chaque variant électrophorétique d'une protéine a été sous le contrôle d'une forme allélique de son gène de structure.

A la différence des allèles des systèmes d'antigènes érythrocytaires, les allèles contrôlant les variants électrophorétiques sont codominants et un individu hétérozygote présente donc deux variants différents détectables par électrophorèse (6). Selon la structure moléculaire de la protéine, chaque variant peut présenter une ou plusieurs bandes. D'autre part, la combinaison de sous-unités moléculaires peut induire la formation de «molécules hybrides » à migration intermédiaire chez un individu hétérozygote, provoquant ainsi l'apparition de nouvelles bandes (6).

La méthode par comptage direct a été utilisée pour le calcul des fréquences alléliques des six systèmes. En effet, les allèles étaient codominants dans ces systèmes et le génotype pouvait donc être déduit directement du phénotype. Par exemple, dans le système $\mathrm{Al}$ comportant les allèles $\mathrm{A}$ et $\mathrm{B}$, les individus $(\mathrm{N}=$ effectif) pouvaient être $\mathrm{AA}$ ou $\mathrm{AB}$ ou $\mathrm{BB}$. La fréquence de l'allèle $\mathrm{A}$ a été calculée selon la formule $\mathrm{qA}=[2(\mathrm{AA})+(\mathrm{AB})] / 2 \mathrm{~N}$ et la fréquence de l'allèle $\mathrm{B}$ selon la formule $\mathrm{qB}=[2(\mathrm{BB})+(\mathrm{AB})] / 2 \mathrm{~N}$. Le test de panmixie vérifiant qu'une population étudiée est en équilibre génétique (loi de Hardy-Weinberg) a été effectué grâce au test statistique du $\chi^{2}$.

\section{RESULTATS ET DISCUSSION}

Les fréquences alléliques des systèmes électrophorétiques $\mathrm{Al}$, Gc, Xk, Es, Tf et Pi pour les 47 poneys étudiés figurent dans le tableau I. Le tableau II représente les phénotypes observés et attendus des quatre systèmes. L'analyse des résultats du test $\chi^{2}$ a indiqué que la population étudiée était en équilibre génétique, les $\chi^{2}$ observés ayant été très en dessous des valeurs seuils du $\chi^{2}$ au-delà desquelles on trouve $\mathrm{P}<0,05$. Pour les loci Tf, Es et Pi, les effectifs théoriques des valeurs inférieures à 5 ont été regroupés pour ne former qu'une seule classe. En procédant de la sorte, les auteurs n'ont pas noté de différence significative entre les phénotypes observés et théoriques à ces systèmes.

Le test de Fisher a été appliqué en comparant les fréquences alléliques obtenues dans la bibliographie chez les chevaux Barbes, Arabes, Arabes-Barbes et le poney de Mogod. Seuls les systèmes électrophorétiques Es et Pi ont montré des différences significatives au test de Fisher $(\mathrm{P}<0,001)$ entre le poney de Mogod et les autres animaux. Ceci peut être dû à la spécificité de ces systèmes chez le poney de Mogod par rapport aux autres races étudiées.

Deux variants du système albumine $\mathrm{A}$ et $\mathrm{B}$ ont été observés. Les fréquences alléliques de ce système (tableau I) se rapprochaient de celles rapportées chez le cheval Barbe de Tunisie (2), Barbe et Arabe-Barbe du Maroc (9). 


\section{Tableau I}

Fréquences alléliques des systèmes électrophorétiques des 47 poneys de Mogod étudiés

\begin{tabular}{|c|c|c|}
\hline & $\begin{array}{c}\text { Système } \\
\text { électrophorétique }\end{array}$ & $\begin{array}{l}\text { Fréquence } \\
\text { allèlique }\end{array}$ \\
\hline \multirow[t]{2}{*}{$\mathrm{Al}$} & A & 0,36 \\
\hline & B & 0,64 \\
\hline Gc & $\mathrm{F}$ & 1 \\
\hline$X k$ & $\mathrm{~K}$ & 1 \\
\hline \multirow[t]{7}{*}{ Tf } & A & 0,02 \\
\hline & $\mathrm{D}$ & 0,41 \\
\hline & $\mathrm{F}$ & 0,01 \\
\hline & F2 & 0,31 \\
\hline & $\mathrm{H}$ & 0,12 \\
\hline & $\mathrm{O}$ & 0,11 \\
\hline & $\mathrm{R}$ & 0,02 \\
\hline \multirow[t]{4}{*}{ Es } & $\mathrm{F}$ & 0,17 \\
\hline & G & 0,18 \\
\hline & 1 & 0,64 \\
\hline & $S$ & 0,01 \\
\hline \multirow[t]{10}{*}{$\mathrm{Pi}$} & $\mathrm{F}$ & 0,02 \\
\hline & 1 & 0,03 \\
\hline & $\mathrm{L}$ & 0,35 \\
\hline & $\mathrm{N}$ & 0,09 \\
\hline & $\mathrm{P}$ & 0,06 \\
\hline & $\mathrm{R}$ & 0,09 \\
\hline & $S$ & 0,09 \\
\hline & $\mathrm{T}$ & 0,01 \\
\hline & U & 0,22 \\
\hline & Z & 0,01 \\
\hline
\end{tabular}

$\mathrm{Al}$ : albumine ; Gc : composant Gc ; Xk : postalbumine ; Tf : transferrine ; Es : estérase basique ; $\mathrm{Pi}$ : préalbumine

Un seul variant de la composante Gc a été détecté chez le poney de Mogod. Il s'agissait du variant F. En revanche, chez le cheval Barbe de Tunisie ou du Maroc et chez le cheval Arabe, le variant S a été observé à des fréquences alléliques faibles $(0,05-0,17)$, sauf dans une étude (1) où $\mathrm{S}$ a été observé à une fréquence élevée chez l'Arabe. L'étude du système Xk a révélé un monomorphisme du système K, alors qu'une étude réalisée sur les chevaux Barbes (3) a montré une fréquence allélique de 0,83 pour le variant $\mathrm{K}$ et de 0,13 pour le variant $\mathrm{S}$. Le tableau III compare les fréquences alléliques de systèmes électrophorétiques rapportées dans la littérature chez les chevaux Barbes, Arabes et Arabes-Barbes.

Les auteurs ont trouvé, chez le poney de Mogod, que la transferrine $\mathrm{R}$ avait une fréquence allélique de 0,02. La plupart des auteurs signalent l'absence du variant $\mathrm{R}$ de la transferrine chez le cheval Arabe (6), sauf Boutouria et Chelbi (1) qui le trouvent à faible fréquence; en revanche, la présence de la transferrine $\mathrm{R}$ chez le cheval Barbe est rapportée par Chabchoub et coll. (3) et Ouragh et coll. (8), et chez l'Arabe-Barbe par Ouragh et coll. (9). Les variants $\mathrm{D}$ et $\mathrm{H}$ de la transferrine ont été trouvés chez la population de poneys de Mogod de la présente étude dans des proportions respectives de 0,41 et 0,12 . Ces proportions demeurent comparables

\section{Tableau II}

Phénotypes observés et attendus des quatre systèmes électrophorétiques étudiés chez les 47 poneys de l'étude

\begin{tabular}{|c|c|c|c|}
\hline & Génotype & Observé & Attendu \\
\hline \multirow[t]{5}{*}{$\mathrm{Al}$} & AA & 5 & 6,15 \\
\hline & $A B$ & 24 & 21,7 \\
\hline & BB & 18 & 19,15 \\
\hline & Total & 47 & \\
\hline & $\chi^{2}$ & 1,05 & $\mathrm{ddl}=2$ \\
\hline \multirow[t]{8}{*}{ Tf } & DD & 7 & 8,09 \\
\hline & DF2 & 15 & 12,03 \\
\hline & $\mathrm{DH}$ & 6 & 4,56 \\
\hline & DO & 2 & 4,15 \\
\hline & $\mathrm{F} 2 \mathrm{~F} 2$ & 4 & 4,47 \\
\hline & Autres & 13 & 13,7 \\
\hline & Total & & \\
\hline & $\chi^{2}$ & 2,53 & $d d l=5$ \\
\hline \multirow[t]{5}{*}{ Es } & Gl & 15 & 10,85 \\
\hline & II & 15 & 19,14 \\
\hline & Autres & 17 & 17,01 \\
\hline & Total & & \\
\hline & $\chi^{2}$ & 2,48 & $d d l=2$ \\
\hline \multirow[t]{5}{*}{$\mathrm{Pi}$} & LL & 9 & 5,79 \\
\hline & LU & 9 & 7,37 \\
\hline & Autres & 29 & 33,8 \\
\hline & Total & & \\
\hline & $\chi^{2}$ & 2,83 & $\mathrm{ddl}=2$ \\
\hline
\end{tabular}

$\mathrm{Al}$ : albumine ; Tf : transferrine ; Es : estérase basique ; Pi : préalbumine

à celles trouvées pour D chez les chevaux Barbes de Tunisie (2) et pour H chez les Barbes au Maroc (8). Kaminski et coll. rapportent que ces variants sont plus fréquents chez les chevaux Arabes que chez les pur-sang Anglais (6). Le variant M de la transferrine a été aussi trouvé chez les Arabes-Barbes du Maroc (9) mais n’a pas été répertorié chez le poney de Mogod.

Chez le poney de Mogod, la prédominance de l'estérase I a été observée malgré la présence des trois autres variants $(\mathrm{F}, \mathrm{G}, \mathrm{S})$. Elle est majoritaire chez les chevaux Arabes-Barbes (8). Chez le cheval Arabe, en revanche, Kaminski et coll. (6) rapportent pratiquement un monomorphisme de l'estérase (6). L'existence du variant $\mathrm{G}$ de l'estérase a été notée chez les poneys étudiés. Ce même variant existe chez le cheval Barbe à un taux comparable. Ouragh et coll. (8), et Kaminski et coll. (6) rapportent qu'il est absent chez le cheval Arabe. Néanmoins, ce variant demeure fréquent chez les chevaux sauvages, les poneys (7), les chevaux de trait et les chevaux demi-sang (6). Ce variant n'a pas été trouvé chez les populations de chevaux Arabes de France avant 1971 (6).

Les variants $\mathrm{H}$ et $\mathrm{G}$ de l'estérase n'ont également pas été trouvés chez les Anglo-Arabes en France. Le variant $\mathrm{S}$ de l'estérase est rare chez les chevaux Arabe (6), Barbe et chez le poney de Mogod. 
Le variant Pi-W des préalbumines n'a pas été trouvé chez le poney Tunisien bien qu'il l'ait été dans un précédent travail (3) chez des chevaux Barbes de Tunisie. Ouragh et coll. (8) le considèrent comme un marqueur génétique du cheval Barbe. Ils affirment qu'on ne peut le trouver, à part chez le cheval Barbe, que chez le cheval Andalou (8). Le variant Pi-W est absent chez le poney de Mogod actuel, mais ceci ne conduit pas à rejeter l'hypothèse d'une relation proche entre le poney de Mogod et le Barbe, car l'allèle a pu être perdu par dérive génétique ; l'allèle n'est d'ailleurs pas à une fréquence très élevée chez le Barbe : 0,14 pour Chabchoub et coll. (3), et 0,04 pour Ouragh et coll. (8). Ces derniers considèrent que les systèmes $\mathrm{Pi}$ et Tf révèlent chez le cheval Barbe un haut niveau de variabilité lié au grand nombre d'allèles qui les constituent.

\section{Tableau III}

Comparaison des fréquences alléliques des systèmes électrophorétiques rapportées dans la bibliographie et obtenues chez les chevaux Barbes, Arabes et Arabes-Barbes

\begin{tabular}{|c|c|c|c|c|c|c|c|}
\hline & \multirow[b]{2}{*}{ Syst. électr. } & \multicolumn{6}{|c|}{ Fréquences alléliques } \\
\hline & & $\begin{array}{c}\mathrm{B} \operatorname{Tn}(3) * \\
\mathrm{~N}=39\end{array}$ & $\begin{array}{l}\text { B Ma (8) } \\
N=206\end{array}$ & $\begin{array}{l}\text { Ar-B (8) } \\
N=594\end{array}$ & $\begin{array}{l}\text { Ar-B (9) } \\
N=348\end{array}$ & $\begin{array}{c}\operatorname{Ar}(7) \\
N=389\end{array}$ & $\begin{array}{c}\operatorname{Ar}(1) \\
N=732\end{array}$ \\
\hline \multirow[t]{2}{*}{$\mathrm{Al}$} & A & 0,27 & 0,3 & 0,3 & 0,3 & 0,25 & \\
\hline & B & 0,73 & 0,7 & 0,7 & 0,69 & 0,75 & \\
\hline \multirow[t]{2}{*}{ Gc } & $\mathrm{F}$ & 0,99 & 0,98 & 0,98 & 0,97 & 0,94 & 0,52 \\
\hline & $\mathrm{S}$ & 0,01 & 0,02 & 0,02 & 0,02 & 0,06 & 0,48 \\
\hline \multirow[t]{2}{*}{$X k$} & K & 0,83 & & & 0,94 & & \\
\hline & $\mathrm{S}$ & 0,17 & & & 0,06 & & \\
\hline \multirow[t]{7}{*}{ Tf } & A & & 0,01 & & 0 & & \\
\hline & $\mathrm{D}$ & 0,44 & 0,3 & 0,26 & 0,26 & 0,09 & 0,15 \\
\hline & $\mathrm{F}$ & & $<0,01$ & 0 & 0,95 & 0,07 & 0,52 \\
\hline & $\mathrm{F} 2$ & 0,24 & 0,36 & 0,4 & 0,39 & 0,6 & \\
\hline & $\mathrm{H}$ & 0,04 & 0,11 & 0,11 & 0,11 & 0,1 & 0,19 \\
\hline & $\mathrm{O}$ & 0,1 & 0,13 & 0,07 & 0,07 & 0,14 & 0,13 \\
\hline & $\mathrm{R}$ & 0,18 & 0,07 & 0,06 & 0,06 & 0 & 0,04 \\
\hline \multirow[t]{6}{*}{ Es } & $\mathrm{F}$ & 0,27 & 0,07 & 0,05 & 0,05 & 0,02 & \\
\hline & G & 0,23 & 0,15 & 0,09 & 0,09 & 0 & \\
\hline & I & 0,47 & 0,71 & 0,82 & 0,82 & 0,98 & \\
\hline & S & 0 & 0,03 & 0,04 & 0,04 & 0 & \\
\hline & $\mathrm{L}$ & 0 & & 0 & & 0 & \\
\hline & $\mathrm{H}$ & 0,03 & 0,02 & 0 & & 0 & \\
\hline \multirow[t]{13}{*}{$\mathrm{Pi}$} & $\mathrm{F}$ & 0,01 & 0,01 & 0,06 & 0,06 & 0,04 & 0 \\
\hline & I & 0,11 & 0,03 & 0,04 & 0,04 & 0,06 & 1 \\
\hline & $L$ & 0,26 & 0,31 & 0,37 & 0,37 & 0,27 & 0 \\
\hline & $\mathrm{N}$ & 0,01 & 0,14 & 0,14 & 0,16 & 0,12 & 0 \\
\hline & $\mathrm{P}$ & 0,12 & 0,01 & 0,02 & & 0,01 & 0 \\
\hline & $\mathrm{R}$ & 0,09 & 0,02 & 0 & 0,02 & 0 & 0 \\
\hline & $S$ & 0,14 & 0,2 & 0,13 & 0,14 & 0,12 & 0 \\
\hline & $\mathrm{T}$ & 0,03 & & 0 & & 0,23 & 0 \\
\hline & U & 0,05 & 0,2 & 0,14 & 0,14 & 0 & 0 \\
\hline & Z & & 0,01 & 0,01 & 0,01 & 0,01 & 0 \\
\hline & $\mathrm{K}$ & 0,01 & 0,01 & 0 & 0,01 & 0 & 0 \\
\hline & W & 0,14 & 0,04 & 0,03 & 0,03 & 0 & 0 \\
\hline & V & & $<0,01$ & $<0,01$ & & 0 & 0 \\
\hline
\end{tabular}

Syst. électr. : systèmes électrophorétiques ; B : cheval Barbe ; Ar : cheval pur-sang Arabe ; Ar-B : cheval Arabe-Barbe ; Tn : Tunisie ; Ma : Maroc

* (1) Boutouria et Chelbi, Maghreb Vét., 1988 ; (3) Chabchoub et coll., El Baytari, 2002 ; (7) Nicolas et Kaminski M., Eperon, 1979 ;

(8) Ouragh et coll., Anim. Genet., 1994 ; (9) Ouragh et coll., Maghreb Vét., 1992

$\mathrm{Al}$ : albumine ; Gc : composant Gc ; Xk : postalbumine ; Tf : transferrine ; Es : estérase basique ; Pi : préalbumine 


\section{CONCLUSION}

L'étude du polymorphisme protéique du sang du poney de Mogod révèle certains points communs avec le cheval Barbe et des différences importantes avec le cheval Arabe et le pur-sang Anglais. C'est notamment le cas du variant D du système transferrine et du variant $\mathrm{G}$ du système estérase.

Il existe néanmoins des différences entre le poney de Mogod et le cheval Barbe : c'est le cas du variant Pi-W qui est présent chez le cheval Barbe et absent chez le poney de Mogod.

Des études plus approfondies appliquées à un nombre plus important de poneys de Mogod mais aussi aux autres chevaux du Maghreb et utilisant d'autres techniques, comme l'analyse des marqueurs microsatellites, sont à réaliser pour une meilleure caractérisation génétique. Elles devraient aboutir à une meilleure connaissance de l'histoire des races et de leurs relations, et à l'estimation de l'effectif efficace de la population de poneys de Mogod. Associée à une meilleure connaissance des spécificités morphométriques et des performances, une caractérisation génétique plus précise permettra de mieux fixer le standard du poney de Mogod et d'éviter les divers croisements intempestifs qui nuiraient à cette race et à la préservation de sa rusticité. La préservation du poney de Mogod permettra d'éviter la disparition d'un patrimoine exceptionnel qui a aidé l'homme à s'implanter dans un environnement de montagne difficile.

\section{BIBLIOGRAPHIE}

1. BOUTOURIA M., CHELBI N., 1988. Les marqueurs génétiques sanguins chez le cheval pur-sang Arabe de Tunisie. Maghreb Vét., 3 : 33-36.

2. CHABCHOUB A., FARHAT E., AMIRA A., LANDOLSI F., TAHRI M., 2000. Le poney de Mogod : contribution à une étude morphologique. El Baytari, 24 : 6-7.

3. CHABCHOUB A., OURAGH L., LANDOLSI F., LASFAR F., 2002. Contribution à une étude du polymorphisme électrophorétique des protéines et enzymes du sang du poney de Mogod et des chevaux Barbes de Tunisie. El Baytari, 28 : 9-10.

4. HOSNI H., 1987. Situation du cheval Barbe en Tunisie. Tous les textes officiels sur le cheval Barbe. Paris, France, Caracole, p. 194.

5. JUNEJA R.K., GAHNE B., SANDBERG K., 1978. Genetic polymorphism of the vitamin $\mathrm{D}$ binding protein and another post-albumin protein in horse serum. Anim. Blood Groups Biochem. Genet., 9: 29-36.
6. KAMINSKI M., URBANSKA-NICOLAS H., 1980. Structure génétique des chevaux Arabes de France: variants électrophorétiques sanguins. Revue Méd. vét., 131 : 613-626.

7. NICOLAS H., KAMINSKI M., 1979. Etude génétique des Anglo-Arabes de France : polymorphisme biochimique sanguin. Eperon (144) : 266-271.

8. OURAGH L., MERIAUX J.-C., BRAUN J.-P., 1994. Genetic blood markers in Arabian, Barb and Arab-Barb horses in Morocco. Anim. Genet., 25: 45-47.

9. OURAGH L., MERIAUX J.-C., BRAUN J.-P., EL KOHEN M., 1992. Les marqueurs génétiques sanguins des chevaux Arabes-Barbes au Maroc. Maghreb Vét., $7: 28-32$.

10. SEBBAG S., 2002. Contribution à l'étude des caractéristiques morphologiques du poney de Mogod en Tunisie. Thèse Doct. vét., université de Sidi-Thabet, Tunisie, 92 p.

Reçu le 13.03.2006, accepté le 26.04.2007

\section{Summary}

Chabchoub A., Mbarki Z., Lasfar F., Landolsi F., Turki I., Ouragh L. Blood Protein Polymorphism in the Mogod Pony in Tunisia

The Mogod pony is native of Northwest Tunisia. Its physical characteristics make him well adapted for mountain use. Since the beginning of the 20th century, its number has greatly decreased. To reverse this trend, the breed will have to be better characterized, in particular from the genetic angle, as this might help search into possible relationships between the pony and horse breeds in the area. Electrophoretic systems of albumin $(\mathrm{Al})$, group specific component (Gc), postalbumin $(X k)$, esterase $(E s)$, transferrin $(\mathrm{Tf})$ and protease inhibitor (Pi) were studied in 47 Mogod ponies by polyacrylamide gel electrophoresis. Allelic frequencies of the six systems were as follows: $\mathrm{Al}^{\mathrm{A}}$ : 0.36; $\mathrm{Al}^{\mathrm{B}}$ : 0.64; $\mathrm{Gc}^{\mathrm{F}}$ : 1.00; $\mathrm{Xk}^{\mathrm{K}}$ : 1.00; $\mathrm{Es}^{\mathrm{l}}$ : 0.64; $\mathrm{Es}^{\mathrm{G}}: 0.18 ; \mathrm{Es}^{\mathrm{F}}: 0.17 ; \mathrm{Es}^{\mathrm{S}}: 0.01 ; \mathrm{Tf}^{\mathrm{D}}: 0.41 ; \mathrm{TfF}^{\mathrm{F}}: 0.31 ; \mathrm{Tf}^{\mathrm{H}}: 0.12$; $\mathrm{Pi}$ : 0.35; $\mathrm{Pi}^{\mathrm{U}}: 0.22 ; \mathrm{Pi}^{\mathrm{R}} ; \mathrm{Pi}^{\mathrm{S}}: 0.09$. The blood protein polymorphism of the Mogod pony was compared with that of thoroughbred Arabian horses, Barb horses and Arab-Barb horses based on bibliographic data.

Keywords: Mogod Pony - Biochemical polymorphism - Blood protein - Tunisia.

\section{Resumen}

Chabchoub A., Mbarki Z., Lasfar F., Landolsi F., Turki I., Ouragh L. Polimorfismo proteico sanguíneo en el potro de Mogod

El potro de Mogod es originario del noroeste de Tunisia. Sus aptitudes físicas le confieren una buena adaptación y utilización en la montaña. Desde el inicio del siglo, los efectivos han sufrido una reducción considerabe. Un plan de relance necesita una mejor caracterización de la raza, principalmente desde el punto de vista genético. Además, esta permitiría la búsqueda de una eventual afiliación de este potro con los otros caballos de la región. Los sistemas electroforéticos albúminas $(\mathrm{Al})$, compuesto $\mathrm{GC}(\mathrm{Gc})$, postalbúminas $(\mathrm{Xk})$, esteres básicos (Es), transferrinas (Tf), pre albúminas (Pi) fueron estudiados en 47 potros Mogod mediante la técnica de electroforesis en gel de poli acrilamida. Las frecuencias alélicas de los seis sistemas fueron estimadas de la manera siguiente: $\mathrm{Al}^{\mathrm{A}}: 0,36 ; \mathrm{Al}^{\mathrm{B}}: 0,64 ; \mathrm{GC}^{\mathrm{F}}: 1,00 ; \mathrm{Xk}^{\mathrm{K}}: 1,00 ; \mathrm{Es}^{\mathrm{l}}: 0,64 ; \mathrm{Es}^{\mathrm{G}}$ : 0,$18 ; \mathrm{Es}^{\mathrm{F}}: 0,17 ; \mathrm{Es}^{\mathrm{S}}: 0,01 ; \mathrm{Tf}^{\mathrm{D}}: 0,41 ; \mathrm{Tf}^{\mathrm{F} 2}: 0,31 ; \mathrm{Tf}^{\mathrm{H}}: 0,12$; $\mathrm{Pi}^{\mathrm{L}}: 0,35 ; \mathrm{Pi}^{\mathrm{U}}: 0,22 ; \mathrm{Pi}^{\mathrm{R}} ; \mathrm{Pi}^{\mathrm{S}}: 0,09$. Una comparación entre el polimorfismo proteico sanguíneo del potro Mogod y de los caballos pura sangre Arabes, Barbes y Arabes-Barbes fue realizada sobre una base bibliográfica.

Palabras clave: Poney Mogod - Polimorfismo bioquímica Proteína sanguínea - Túnez. 